\title{
Téoros
}

Revue de recherche en tourisme

\section{Les associations sont aussi des entreprises}

\section{Normand Dulude}

Volume 5, numéro 3, novembre 1986

La gestion touristique : les nouvelles technologies

URI : https://id.erudit.org/iderudit/1080540ar

DOI : https://doi.org/10.7202/1080540ar

Aller au sommaire du numéro

Éditeur(s)

Université du Québec à Montréal

ISSN

0712-8657 (imprimé)

1923-2705 (numérique)

Découvrir la revue

Citer cet article

Dulude, N. (1986). Les associations sont aussi des entreprises. Téoros, 5(3),

34-42. https://doi.org/10.7202/1080540ar d'utilisation que vous pouvez consulter en ligne.

https://apropos.erudit.org/fr/usagers/politique-dutilisation/ 


\title{
Les associations sont aussi des entreprises
}

\author{
par Normand Dulude*
}

Dans le domaine du tourisme, plus particulièrement chez les entreprises de tourisme commercial, les associations gestionnaires d'équipements récréotouristiques ont fort mauvaise réputation: mauvaise gestion, absence de souci de rentabilité, concurrence déloyale résultant de l'aide gouvernementale qui leur est accordée. Pourtant, et nous allons tenter de le démontrer, les associatins sont aussi des entreprises qui, à bien des égards, se comparent à celles du secteur privé à but lucratif.

Notre contribution n'a pas la prétention d'analyser de façon systématique toutes les facettes de la gestion des organismes du secteur associatif. L'objet de notre contribution est plus modeste et consiste, en un premier temps, à mieux cerner la réalité économique du secteur associatif, dans un deuxiême temps, à dégager ce qui, à notre avis, distingue ce secteur de celui du tourisme commercial, et, enfin, à examiner un des aspects qui nous apparaît primordial pour l'avenir de ce secteur, le financement. Notre expérience de plus de dix ans auprès du secteur associatif nous a aidé considérablement à livrer ce constat et ces pistes de développement.

\section{Le tourisme associatif: de quoi parlons-nous?}

La réalité sociale du tourisme québécois remonte a la fin du XIXe siecle avec la création des premières colonies de vacances. On connaitt le succès que remportèrent ces colonies gérées en grande partie par le clergé et dont le nombre a atteint plusieurs centaines à travers le Québec. La laïcisation de ce mouvement au temps de la révolution tranquille les a fait se transformer en cams de vacances, diversifiant les clientèles et s'adaptant même aux besoins de vacances des familles.

C'est dans les années ' 30 que naissaient, dans le courant international, les premières auberges de jeunesse au Québec. Cellesci se développèrent par la suite grâce à l'intervention des gouvernements fédéral et provincial au cours des années ' 60 .

* Normand Dulude est consultant en développement organisationnel Loisirs et Tourisme.
Les premiers camps familiaux sont apparus dans les années ' 40 dans le cadre de la Ligue Ouvrière Catholique, qui tenta les premières expériences de camps ouvriers de vacances.

Les pratiques sociales de tourisme, aujourd'hui mises de I'avant par le secteur du tourisme associatif, remontent à bien des années et sont issues de la volonté des milieux concernés de faire en sorte que les vacances et le tourisme soient accessibles à tous.

L'importance de l'activité économique générée par l'ensemble des équipements du secteur associatif doit être signalée. Nous allons présenter un certain nombre de données pour dếmontrer cette importance.

Ainsi, l'ensemble des réseaux du tourisme associatif ont réalisé en 1985-86 plus de deux millions sept cent mille (2 700 000) nuitées, rejoint plus de cinq cent mille (500 000) personnes. De plus, les équipements de ces réseaux ont généré plus de soixante-treize millions de dollars (73000 000 \$) en opération qui, pour la plupart, sont réinvestis dạs l'économie régionale environnante ${ }^{(1)}$. Le nombre des emplois ainsi créés est également non négligeable. Comme seul exemple, l'Association des Camps évalue à plus de 4000 le nombre d'emplois saisonniers crées par ses 111 camps membres. De façon globale, le secteur associatif a créé cette mểme année 112190 emplois/semaine ${ }^{(1)}$. Comme le soulignait le Ministre Picotte, à l'ouverture de la conférence sectorielle sur le loisir touristique, en avril dernier, l'importance économique de ces réseaux ne fait pas de doutew.

Produisant des biens et services dans le domaine du tourisme, qui représentent grosso modo $5 \%$ des dépenses directes reliées à la pratique des activités de loisirs des québécoises et des québécois, le secteur associatif représente une part modeste mais tout de même significative de l'économie du Québec ${ }^{(1)}$.

C'est l'ensemble de ces données qui doivent être prises en compte pour mieux analyser sur I'investissement que fait le gouvernement en subventionnant partiellement ces réseaux. En effet, avec une aide de l'État d'environ 4 millions de dollars. le secteur associatif aura généré des retombées économiques de plus de 146 millions $\$$, si l'on utilise un taux pour l'effet multi= plicateur de $2,0^{(1)}$, taux très conservateur si on le compare à ceux utilisés pour certains secteurs touristiques traditionnels ${ }^{(1)}$.

Pour fins de comparaison, mème si elle peut sembler boîteuse et malgré le peu de crédit accordé actuellement à ce genre d'exercice, M. Yves Michaud, pdg du Palais des Congrès de Montréal, annonçait récemment que son organisme avait généré des retombées économiques de 95 millions de dollars en regard d'un déficit d'opération de 7 millions $\$$, assumé en totalité par le gouvernement du Québec. Le secteur associatif pourrait aussi démontrer que l'investissement de l'État dans ses activités est rentable.

Il faut souligner qu'au-delà de cette importance économique, les objectifs d'accessibilité demeurent au centre des préoccupations des gestionnaires de ces equipements, qui doivent allier cette accessibilité et l'équilibre financier nécessaire au maintien et au développement de leur entreprise.

\section{Ce qui distingue le secteur associatif}

Il n'est évidemment pas possible de prétendre effectuer ici une présentation exhaustive du caractère distinct des associatons en regard des entreprises commerciales.

Certaines association n'y retrouveront pas toutes les caractéristiques qui collent à leur réalité, d'autres ne se sentiront pas concernées par tous les éléments ici esquissés. Qu'on veuille bien nous en excuser, ce choix ne résultant pas d'un jugement de valeur mais plutôt d'un souci de présentation générale, donc simplifiée et nécessairement incomplète.

\section{D'abord et avant tout: les structures}

Fondant ses activités et ses programmes sur la nécessaire satisfaction de ses membres, 
I'entreprise associative se distingue d'abord et avant tout par ses structures.

En effet, les différences, à ce niveau, touchent:

- l'existence de membres plutôt que celle d'actionnaires;

- la liberté d'adhésion et de retrait des membres:

- l'absence de protit individuel pour les membres;

- l'existence de structures de gestion démocratique;

- la non-distribution aux membres des surplus d'opération.

Le cadre législatif de corporation sans but luctatif y est pour beaucoup dans ces caractéristiques. Si la nécessité d'un équilibre budgétaire demeure une préoccupation constante pour ses gestionnaires, aucune recherche de profit ne vient orienter l'offre touristique des associations, qui se veut la plus adaptée possible aux besoins des clientèles rejointes et des membres.

\section{Une ressource spécifique: le travail bénévole}

Les objectifs d'accessibilité sont maintenus d'abord et avant tout grâce au travail incessant des bénévoles qui oeuvrent au sein des associations gestionnaires.

Dans les éléments distinctifs, en terme de gestion, entre les associations et les entreprises commerciales, cette participation des bénévoles est sans doute le principal élé:ment à retenir.

En effet, la production des services des associations repose en grande partie sur cet apport du bénévolat qui permet à celles-ci de fonctionner et d'offrit des prestations accessibles. En regard de cet apport, la contribution gouvernementale est somme toute

\section{Le titre associatif}

Il s'agit essentiellement de permettre aux associations sans but lucratif de se créer des fonds pour l'investissement en mettant à leur disposition la possibilité d'émettre ce qu'en France on a appelé le titre associatif, c'est-à-dire des actions privilégiées propres aux associations. En effet, les associations gestionnaires ne peuvent avoir recours, selon la loi actuelle, à une émission de capital sous forme d'actions privilégiées comme le font les entreprises à but lucratif; les possibilités de financement sont: l'emprunt sur les marchés financiers normaux ou l'aide de l'État. L'on comprendra aisément que celui-ci soit si souvent considéré, dans le système actuel, comme la seule ressource pour la consolidation et le développement des équipements de tourisme associatif moins importante que l'on est porté à la croire.

Le travail bếnếvole coexiste avec le travail de salariés qui s impliquent dans les associations non seulement à titre professionnel mais aussi souvent à titre de militant, avec tout ce que cela suppose au plan d'une rémunération moindre par rapport à la juste valeur du travail accompli. Se posent aussi, dans ce contexte, les problèmes de cohabitation entre salariés et bénévoles au plan du partage des responsabilites, que l'on ne retrouve pas nécessairement pas dans le secteur commercial. Cette coexistence n'est pas toujours sans heurt, les salariés reprochant quelquefois aux bénévoles leur manque de professionnalisme et ces derniers reprochant aux salariés leur manque de motivation et d'engagement.

Ce problème est d'autant plus complexe que la distinction entre bénévolat et emploi salarié n'est pas toujours claire: du bénévole sans aucune rémunération au permanent d'association qui n'adhère pas à ses objectifs, il y a toutes les variantes possibles. Diverses structures de cogestion ont été expérimentées au sein du secteur associatif, afin de régler si tant faire se peut, ce problème de partage de responsabilités. Ces expériences ne peuvent toutefois pas être considérées comme représentatives des orientations des associations au plan de la gestion. Il n'en reste pas moins que la clarification des mandats et des apports de chacun au sein d'une association demeure un des problèmes importants auxquels ont à faire face les associations gestionnaires.

Il serait important, et des travaux seront entrepris à ce niveau, de mieux connaître le travail bénévole au sein des associations pour mieux en situer son apport au plan économique et social. On ne peut avoir une idée juste de la productivité d'une association si on ne tient pas compte de cet apport de travail non-rémunéré.

En permettant aux associations gestionnaires d'avoir recours à des titres associatifs, le gouvernement pourrait faciliter les investissements dans ce secteur. Le gouvernement pourrait par exemple garantir auprès des épargnants leurs éventuels investissements et, plutót que de contribuer pour la totalité des fonds nécessaires à la consolidation, payer les intérêts à verser sur les titres associatifs.

Il faut bien comprendre que cette hypothèse ne peut être considérée comme la panacée à tous les problèmes de financement qu'ont à subir les associations gestionnaires. Il ne s'agit, comme nous l'avons dit plus haut, que d'une solution complémentaire à la nécessaire implication du gouvernement dans l'accessibilité au tourisme et aux vacances.

\section{Les associations à I'heure du financement}

Les différences les plus marquées entre le secteur associatif et le secteur commercial se situent sans aucun doute au plan du financement.

La population québécoise demande d'avoir accềs à des équipements qui, tout en offrant une animation et des activités correspondant à ses besoins, sont en mesure de lui garantir une infrastructure d'hébergement sécuritaire, de bon confort et répondant à ses besoins d'intimité. Le secteur du tourisme associatif a, dans ce sens, des besoins de consolidation évidents.

Dans la mesure où les associations gestionnaires fonctionnent de plus en plus comme des entreprises, elles ont des besoins financiers croissants, à court et long terme. Tout le probleme du financement, non seulement en termes d'opérations courantes mais aussi et surtout en termes d'investissement nécessaire à la consolidation et au développement, se pose de façon cruciale.

Au niveau des opérations courantes, jusqu'à maintenant, les subventions constituaient un apport financier régulier au plan du fonctionnement. Si ce support diminue de façon tangible et ce, depuis déjà quelques années, les gestionnaires d'associations n'ont d'autres choix que d'augmenter les couts des prestations pour couvrir les besoins financiers, mettant à sérieuse épreuve leurs objectifs d'accessibilité. Si les cotisations des membres représentent en thérie une autre source de financement, celles-ci ne couvrent en fait qu'une faible partie de ces besoins financiers. Les gestionnaires comptent également sur l'appui du secteur privé (fondations, commandites, etc.) pour contribuer à l'accessibilité de leurs équipements et ainsi compenser lo plus possible le retrait progressif de l'Etat.

Au niveau des investissements nécessaires à la consolidation et au développement, le problème du financement se pose avec plus d'acuité. En effet, les possibilités d'autofinancement sont limitées à cause du caractère sans but lucratif de l'entreprise. Celle-ci n'ayant pas comme objectif de faire des profits mais plutôt de rendre ses équipements les plus accessibles possibles, done de réduire au maximum les tarifs, $n$ "a pu se créer de fonds propres qui pourraient lui permettre d'investir elle-mème dans son établissement.

C'est cette épineuse question qui est soulevée présentement par le secteur associatif auprès du Gouvernement du Québec. quand il revendique une nécessaire refonte des programmes d'aide, permettant aux associations de consolider leurs équipements et de continuer à répondre à leurs objectifs.

(Suite à la page 42) 
Nous avons privilégié certains territoires à I'exclusion d'autres, en fonction de frontières sociales bien détinies, Excluant tous les secteurs touristiques de la rive sud du Fleuve Saint-Laurent, I'espace à étudier correspond globalement à trois secteurs touristiques de la région mauricienne: $\mathrm{A}$ Trois-Rivières, B- Le chemin du Roy, et C- La vallée du Saint-Maurice. Ce territoire est délimité au sud par la rive nord du Fleuve Saint-Laurent, au nord par la ville de La Tuque, et s'êtend d'est en ouest entre les $72^{\circ}$ et $73^{\circ}$ de longitude.

\section{La Mauricie comme espace géographique et touristique}

Espace géographique et touristique la Mauricie doit aussi etre vue dans son entite sociale, et dans les rapports sociaux qu'elle engendre. Ainsi, avant de proposer tout projet d'aménagement, nous croyons qu'il est utile d'analyser le phénomène des fêtes populaires, comme pratique touristique a trois points de vue: 1 . de la circulation des réseaux sociaux internes à la rếgion qui débordent vers l'extérieur (et vice versa); 2 . de la reproduction des patrimoines familiaux; et 3. de l'échange des biens matếriels et immatériels. Ce dernier point de vue est essentiel à notre démarche, car beaucoup de fêtes ne sont explicables que dans le contexte de la reproduction (economique, politique et religieuse) des groupes.

Bref, si on veut definir la Mauricie comme un espace touristique régional, économiquement exploitable de ce point de vue, il ne faut pas négliger avant tout de la considérer comme un espace social en soi, où les membres et anciens membres (incluant sur le plan symbolique les ancêtres-morts) de chaque communauté locale et parentale, envahissent cet espace pendant ces périodes festives, circulent à l'intérieur de leurs frontières pour echanger des visites à leurs parents, amis et voisins, ou les débordent pour justement fuir la fête. Par ailleurs, si le contexte historique des fêtes populaires en Mauricie favorise de grands rassemblements et augmenta le nombre de ces ếvếnemenis depuis vingt ans, on peut penser aussi, que c'est gräce ă l'extension des réseaux de relations inter-localités et interfamiliales, en vue de la reproduction des groupes qui la composent, que de telles réunions sont possibles.

Afin de rétablir les faits concernant ces grandes concentrations, nous allons mettre l'accent sur l'étude des réseaux sociaux spécifiques qui rayonnent à partir d'un point central localisé à Sainte-Tite. En s'interrogeant sur la elientèle touristique qui fréquente cette ville, pendant la période du Festival Western, des rapports qu'elle entretient avec cette population locale, nous nous demanderons alors si les habitants de Saint-Tite echangent hl leur tour des visites et assistent à d'autres fêtes ailleurs, et oc en fonction des réseaux que nous aurons découvert. Nous vérifierons, ensuite ce modèle des réseaux en l'appliquant à chacune des localités à l'étude.

Sur le plan économique, l'industrie touristique, d'après I'A.T.R. du Coeur-duQuébec: "tient un rôle secondaire, de support et d'appui" par rapport aux autres activités economiques de la région. (2) Mais admettons quand meme que l'argent investi depuis une vingtaine d'années, en Mauricie, dans l'aménagement de la nature comme site de villégiature (ex: le Parc National de la Mauricie, la Réserve Faunique du Saint-Maurice, les ZECS, etc.). de loisirs, de chasse et de pëche a de toute evidence aidé cortains groupes à se refaire, à court terme, un capital politique et financier.

\section{Fêtes et dimension économique}

Situées à proximité de ces sites, les localités où se déroulent les fëtes profitent des touristes qui arrêttent au passage. La féte populaire fait done partie du circuit touristique. Dépendantes en grande partie des politiques de subventions gouvernementales, des nombreux commanditaires (brasseries, caisses populaires, plusieurs commerces locaux, etc.), les fètes populaires s'insèrent dans une mème visée économique et politique: attirer de plus en plus de touristes pour promouvoir l'exploitation de certaines ressources naturelles (I'orignal, le poulamon), vendre certains produits locaux (le cuir, le bois, les canots, le blé d'Inde, l'artisanat, etc.), les produits nationaux et d'importation, et aussi pour louer toute sorte d'equipement. En fait, derrière chaque promotion publicitaire se cache une certaine idéologie mercantile qui incite les touristes à dépenser pour mieux fêter. Ce qui nous intéresse dans cette dernière question, c'est l'étude des représentations que le touriste-fetard-consommateur accorde à la valeur de l'argent en ces périodes de festivites; et d'autre part, l'aspect positif de la fête qui anéantit ou neutralise toute critique négative à l'égard de ceux qui font des profits avec l'argent des fetards. Ceux-ci profitent aussi, dans une certaine mesure, de ceux-là car ils font partie de la fête. Par exemple, les compagnies de bières investissent beaucoup d'argent dans ces festivités et à voir les centaines de millier de gallons de bières qui se boivent lors de ces événements, on constate une certaine relation de complicité entre le brasseur et le buveur. Ce type de complicité vaut aussi pour les autres vendeurs de fétes: boutiquiers d'accessoires festifs, d'artisanats, de gadgets variés; les restaurants de bouffe träditionnelle, de fast food, et la bouffe qu'on trouve seulement dans ces occasions.

Finalement, nous essaierons de voir dans quelle mesure la Mauricie se donne des airs de fêtte à l'américaine. Le cas apparcnt du Festival Western de Saint-Tite est la première cible visce par les critiques. Cepen- dant, du point de vue de l'analyse, nous pensons que le décor est une forme de simulacre: il peut être américain, russe, papou, voir même québécois décrivant un patrimoine passéiste, mais l'expression festive profonde, demeure authentique en son fond populaire. Cette résistance se traduit surtout par des éléments d'une tradition fortement empreinte de la religiosité primitive. Ce qui se vit sur la place publique déborde largement les cadres imposés de l'extérieur ct même ceux proposés par l'organisation officielle locale. Il ne faut pas nier toutefois certaines transformations historiques qui sont survenus aux cours des siècles.

$f$

(1) Ce document est une brochure publiée par le Ministẻre du Tourisme en collaboration avec I'Association Touristique lou A.T.R.) du "Coeur-du-Quebesc", 2e ediction, 1986.

(2) CAZELAIS, Normand: "Rible et action des associations fowristiques régionales" in Teroros, Vol. 5, no. 1, mars 1996, p. 31

Les associations

(Suite de la page 35)

Cette refonte des programmes d'aide, que le gouvernement tarde à entreprendre, devrait, à notre avis, être l'occasion d'examiner également les alternatives, ou plutôt les compléments, à l'aide gouvernementale.

Ces alternatives ou compléments pourraient, par exemple, viser à mobiliser une épargne qui n"emprunterait pas les circuits traditionnels de placement. Les experriences européennes comme celle, récente, du titre associatif, nous apparaissent intéressantes à examiner.

Chose certaine, l'Etat aura toujours une responsabilitế à assumer dans le secteur du tourisme associatif si l'on veut que l'aceessibilite aux vacanos et au touristhe soit une réalité au Québec. Les formes d'aide employées jusqu'ici n'ont pas contribué, à notre avis, à développer un secteur hautement performant au plan de la gestion, mème s'il peut se comparer avantageusement à d'autres secteurs touristiques traditionnels. Une refonte des programmes d'aide devra avoir comme préoceupation cette nécessaire performance.

Les moyens qu'il faudra mettre de l'avant pour assurer la consolidation et le développement du secteur associatif devront également tenir compte d'un nécessaire maintien de sa spécificité. Partic intégrante de l'économie touristique du Québec, le secteur associatif doit demeurer le principal animateur d'un tourisme accessible offrant des prestations de qualité à des prix abordables. Qui d'autres pourraient jouer ce rôle? $f$

\section{Notes}

(1) II s'agit ici d'hypotheses de travail. 